\title{
Rings whose total graphs have small vertex-arboricity and arboricity
}

\author{
Morteza Fatehi (iD), Kazem Khashyarmanesh*(D), Abbas Mohammadian (D) \\ Department of Pure Mathematics, Ferdowsi University of Mashhad, P.O.Box 1159-917r5, Mashhad, Iran
}

\begin{abstract}
Let $R$ be a commutative ring with non-zero identity, and $Z(R)$ be its set of all zero-divisors. The total graph of $R$, denoted by $T(\Gamma(R))$, is an undirected graph with all elements of $R$ as vertices, and two distinct vertices $x$ and $y$ are adjacent if and only if $x+y \in Z(R)$. In this article, we characterize, up to isomorphism, all of finite commutative rings whose total graphs have vertex-arboricity (arboricity) two or three. Also, we show that, for a positive integer $v$, the number of finite rings whose total graphs have vertex-arboricity (arboricity) $v$ is finite.
\end{abstract}

Mathematics Subject Classification (2020). Primary: 05C99, Secondary: 13A99

Keywords. total graph, arboricity, vertex-arboricity

\section{Introduction}

In [1], D.F. Anderson and A. Badawi introduced the total graph of ring $R$, denoted by $T(\Gamma(R))$, as the graph with all elements of $R$ as vertices, and for distinct $x, y \in R$, the vertices $x$ and $y$ are adjacent if and only if $x+y \in Z(R)$, where $Z(R)$ is the set of zero-divisors of $R$. They studied some graph theoretical parameters of $T(\Gamma(R))$ such as diameter and girth. In addition, they showed that the total graph of a commutative ring is connected if and only if $Z(R)$ is not an ideal of $R$. In [7], H.R. Maimani et al. gave the necessary and sufficient conditions for the total graphs of finite commutative rings to be planar or toroidal and in [5] T. Chelvam and T. Asir characterized all commutative rings such that their total graphs have genus two.

Suppose that $G$ is a graph, and let $V(G)$ and $E(G)$ be the vertex set and edge set of $G$, respectively. The vertex-arboricity of a graph $G$, denoted by $v a(G)$, is the minimum positive integer $k$ such that $V(G)$ can be partitioned into $k$ sets $V_{1}, V_{2} \ldots, V_{k}$ such that $G\left[V_{i}\right]$ is a forest for each $i \in\{1,2, \ldots, k\}$, where $G\left[V_{i}\right]$ is the induced subgraph of $G$ whose vertex set is $V_{i}$ and its edge set consists of all of the edges in $E(G)$ that have both endpoints in $V_{i}$. This partition is called acyclic partition. The vertex-arboricity can be viewed as a vertex coloring $f$ with $k$ colors, where each color class $V_{i}$ induces a forest; namely, $G\left[f^{-1}(i)\right]$ is an acyclic graph for each $i \in\{1,2, \ldots, k\}$. Vertex-arboricity, also known as point arboricity, was first introduced by G. Chartrand, H.V. Kronk, and C.E.

\footnotetext{
*Corresponding Author.

Email addresses: m.fatehi.h@gmail.com (M. Fatehi), khashyar@ipm.ir (K. Khashyarmanesh), abbasmohammadian1248@gmail.com (A. Mohammadian)

Received: 10.07.2019; Accepted: 03.05.2020
} 
Wall [4] in 1968. Note that a graph with no cycles is a forest, and it has vertex-arboricity one.

Likewise, the arboricity of a graph $G$, denoted by $\nu(G)$, is the least number of linedisjoint spanning forests into which $G$ can be partitioned, that is, there is some collection of $\nu(G)$ subgraphs of $G$, where each subgraph is a forest and each edge in $G$ is in exactly one such subgraph. Arboricity of a graph was first introduced by C. St. J. A. Nash-Williams [4] in 1964.

The main purpose of this paper is to characterize all finite commutative rings whose total graph has vertex-arboricity (arboricity) two or three. In addition, we show that, for a positive integer $v$, there are only finitely many finite rings whose total graph has vertex-arboricity (arboricity) $v$.

Now, we recall some definitions of graph theory which are necessary in this article. Let $G=(V(G), E(G))$ be a graph with vertex set $V(G)$ and edge set $E(G)$. We use $n$ and $e$ to denote the number of vertices and the number of edges of $G$, respectively. A graph in which each pair of distinct vertices is joined by an edge is called a complete graph. We use $K_{n}$ to denote the complete graph with $n$ vertices. A bipartite graph $G$ is a graph whose vertex set $V(G)$ can be partitioned into two subsets $V_{1}$ and $V_{2}$ such that the edge set of such a graph consists of precisely those edges which join vertices in $V_{1}$ to vertices of $V_{2}$. In particular, if $E(G)$ consists of all possible such edges, then $G$ is called the complete bipartite graph and denoted by the symbol $K_{r, s}$, where $\left|V_{1}\right|=r$ and $\left|V_{2}\right|=s$. For a vertex $x \in V(G), \operatorname{deg}(x)$ is the degree of vertex $x, \delta(G)=\min \{\operatorname{deg}(x): x \in V(G)\}$, $\Delta(G)=\max \{\operatorname{deg}(x): x \in V(G)\}$. For a nonnegative integer $d$, a graph is called $d$-regular if every vertex has degree $d$. Let $S \subset V(G)$ be any subset of vertices of $G$. Then the induced subgraph $G[S]$ is the graph whose vertex set is $S$ and whose edge set consists of all of the edges in $E(G)$ that have both endpoints in $S$. A spanning subgraph for $G$ is a subgraph of $G$ which contains every vertex of $G$. A graph without any cycle is called acyclic graph. A forest is an acyclic graph. Let $G_{1}$ and $G_{2}$ be subgraphs of $G$, we say that $G_{1}$ and $G_{2}$ are disjoint if they have no vertex and no edge in common. The union of two disjoint graphs $G_{1}$ and $G_{2}$, which is denoted by $G_{1} \cup G_{2}$ is a graph with $V\left(G_{1} \cup G_{2}\right)=V\left(G_{1}\right) \cup V\left(G_{2}\right)$ and $E\left(G_{1} \cup G_{2}\right)=E\left(G_{1}\right) \cup E\left(G_{2}\right)$. For any graph $G$, the disjoint union of $k$ copies of $G$ is denoted by $k G$. Graphs $G$ and $H$ are said to be isomorphic to one another, written $G \cong H$, if there exists a one-to-one correspondence $f: V(G) \rightarrow V(H)$ such that for each pair $x, y$ of vertices of $G, x y \in E(G)$ if and only if $f(x) f(y) \in E(H)$. Also, for a rational number $p,\lceil p\rceil$ is the first integer number greater than or equal to $p$, and $\lfloor p\rfloor$ is the first integer number less than or equal to $p$.

\section{Basic properties}

First of all, let us recall some of the basic facts about total graphs and vertex arboricity, which we shall use in the rest of the paper.

Lemma 2.1 ([7, Lemma 1.1]). Let $x$ be a vertex of $T(\Gamma(R))$. Then the following statements are true.

(i) If $2 \in Z(R)$, then $\operatorname{deg}(x)=|Z(R)|-1$.

(ii) If $2 \notin Z(R)$, then $\operatorname{deg}(x)=|Z(R)|-1$ for every $x \in Z(R)$ and $\operatorname{deg}(x)=$ $|Z(R)|$ for every vertex $x \notin Z(R)$.

Remark 2.2. It is clear that $v a(G)=1$ if and only if $G$ is acyclic. For a few classes of graphs, the vertex-arboricity is easily determined. For example, $v a\left(C_{n}\right)=2$, where $C_{n}$ is a cycle graph with $n$ vertices. If $n$ is even, $v a\left(K_{n}\right)=\frac{n}{2}$; while if $n$ is odd, $v a\left(K_{n}\right)=\frac{n+1}{2}$. So, in general, $v a\left(K_{n}\right)=\left\lceil\frac{n}{2}\right\rceil$. Also, $v a\left(K_{r, s}\right)=1$ if $r=1$ or $s=1$, and $v a\left(K_{r, s}\right)=2$ otherwise. 
Lemma 2.3 ([3, Lemma 1]). Let $G$ be the disjoint union of graphs $G_{1}, G_{2}, \ldots, G_{k}$. Then, for all $i$ with $1 \leq i \leq k$,

$$
v a(G)=\max v a\left(G_{i}\right) .
$$

Now, we are ready to show that for a positive integer $v$, there are only finitely many finite rings whose total graph has vertex-arboricity $v$.

Theorem 2.4. For any positive integer $v$, the number of finite rings whose total graphs have vertex-arboricity $v$ is finite.

Proof. Let $R$ be a finite ring. We want to obtain a complete subgraph (with vertex set $T)$ of $T(\Gamma(R))$. To achieve this, we consider the following two cases:

(a) $R$ is local. In this case $Z(R)$ is the maximal ideal of $R$ and $|R| \leq|Z(R)|^{2}$ [8]. In this situation, we put $T=Z(R)$.

(b) $R$ is not local. Then there is a natural number $n \geq 2$ and there are local rings $R_{1}, R_{2}, \ldots, R_{n}$ such that $R=R_{1} \times R_{2} \times \cdots \times R_{n}$. We may assume that $\left|R_{1}\right| \leq\left|R_{2}\right| \leq$ $\cdots \leq\left|R_{n}\right|$. Now put $R_{1}^{*}=0 \times R_{2} \times \cdots \times R_{n}$. Since $|R|=\left|R_{1}\right|\left|R_{1}^{*}\right|$, we have $|R| \leq\left|R_{1}^{*}\right|^{2}$. In this situation, we put $T=R_{1}^{*}$.

Now, it is easy to see that, for every elements $x$ and $y$ of $T, x$ is adjacent to $y$ in $T(\Gamma(R))$. Thus there is an induced subgraph $K_{|T|}$ in $T(\Gamma(R))$. Hence Remark 2.2 implies that $v a\left(K_{|T|}\right) \leq v$, and so $\left\lceil\frac{|T|}{2}\right\rceil \leq v$. Thus $|R| \leq 4 v^{2}$, and so the proof is complete.

Let $\operatorname{Reg}(\Gamma(R))$ be the induced subgraph of $T(\Gamma(R))$ with vertices $\operatorname{Reg}(R)=R-Z(R)$, and $Z(\Gamma(R))$ be the induced subgraph of $T(\Gamma(R))$ with vertices $Z(R)$. Next, we record some facts concerning total graphs. If $Z(R)$ is an ideal of $R$, then $Z(\Gamma(R))$ is a complete subgraph of $T(\Gamma(R))$ and is disjoint from $\operatorname{Reg}(\Gamma(R))$. Thus, the following theorem of D.F. Anderson and A. Badawi gives a complete description of $T(\Gamma(R))$.

Theorem 2.5 ([1, Theorem 2.2]). Let $R$ be a commutative ring such that $Z(R)$ is an ideal of $R$, and let $|Z(R)|=n$ and $\left|\frac{R}{Z(R)}\right|=m$. Then the following statements hold.

(i) If $2 \in Z(R)$, then $\operatorname{Reg}(\Gamma(R))$ is the union of $m-1$ disjoint $K_{n}$ 's.

(ii) If $2 \notin Z(R)$, then $\operatorname{Reg}(\Gamma(R))$ is the union of $\frac{m-1}{2}$ disjoint $K_{n, n}$ 's.

Theorem 2.6. Let $R$ be a finite commutative ring with identity and $I$ be a nontrivial ideal contained in $Z(R)$. Set $|I|=n$ and $\left|\frac{R}{I}\right|=m$. Then the following statements hold.

(i) If $2 \in I$, then $v a(T(\Gamma(R))) \geq\left\lceil\frac{n}{2}\right\rceil$.

(ii) If $2 \notin I$, then $v a(T(\Gamma(R))) \geq \max \left\{\left\lceil\frac{n}{2}\right\rceil, 2\right\}$.

Proof. Let $G$ be the spanning subgraph of $T(\Gamma(R))$ such that, for every two vertices $x, y \in R, x$ is adjacent to $y$ in $G$ if $x+y \in I$. Now, since $I$ is an ideal of $R$ contained in $Z(R)$, by making obvious modification to the proof of Theorem 2.5 , one can show that

$$
G=\left\{\begin{array}{lr}
m K_{n} & \text { if } 2 \in I \\
K_{n} \bigcup\left(\frac{m-1}{2}\right) K_{n, n} & \text { if } 2 \notin I .
\end{array}\right.
$$

Now, by Remark 2.2 in conjunction with Lemma 2.3 , we have the following equalities

$$
v a(G)= \begin{cases}\left\lceil\frac{n}{2}\right\rceil & \text { if } 2 \in I \\ \max \left\{\left\lceil\frac{n}{2}\right\rceil, 2\right\} & \text { if } 2 \notin I .\end{cases}
$$

Now, since $G$ is a subgraph of $T(\Gamma(R))$, we have that $v a(G) \leq v a(T(\Gamma(R)))$, and so the proof is complete.

The following corollary is immediate from Theorem 2.5.

Corollary 2.7. Let $R$ be a finite commutative ring with identity, $Z(R)$ be nontrivial ideal of $R$ and set $|Z(R)|=n$ and $\left|\frac{R}{Z(R)}\right|=m$. Then the following statements hold.

(i) If $2 \in Z(R)$, then $v a(T(\Gamma(R)))=\left\lceil\frac{n}{2}\right\rceil$.

(ii) If $2 \notin Z(R)$, then $v a(T(\Gamma(R)))=\max \left\{\left\lceil\frac{n}{2}\right\rceil, 2\right\}$. 


\section{The vertex-arboricity of the total graph}

For any graph $G$, the girth of $G$, denoted by $\operatorname{gr}(G)$, is the length of a shortest cycle in $G(\operatorname{gr}(G)=\infty$ if $G$ contains no cycles). The following Theorem of Anderson and Badawi implies that $T(\Gamma(R))$ has vertex-arboricity one if and only if either $R$ is an integral domain or $R$ is isomorphic to $\mathbb{Z}_{4}$ or $\frac{\mathbb{Z}_{2}[x]}{\left(x^{2}\right)}$.

Theorem 3.1 ([2, Theorem 4.7]). Let $R$ be a commutative ring. Then $\operatorname{gr}(T(\Gamma(R))) \in$ $\{3,4, \infty\}$. Moreover,

(i) $\operatorname{gr}(T(\Gamma(R)))=\infty$ if and only if either $R$ is an integral domain or $R$ is isomorphic to $\mathbb{Z}_{4}$ or $\frac{\mathbb{Z}_{2}[x]}{\left(x^{2}\right)}$

(ii) $\operatorname{gr}(T(\Gamma(R)))=4$ if and only if $R$ is isomorphic to $\mathbb{Z}_{2} \times \mathbb{Z}_{2}$, and

(iii) $\operatorname{gr}(T(\Gamma(R)))=3$ otherwise.

Now, we will classify, up to isomorphism, all finite commutative rings whose total graphs have vertex-arboricity two or three. We begin with a following result which is essentially due to Raghavendran.

Theorem 3.2 ([10, Theorem 2]). Let $R$ be a finite commutative local ring with nonzero identity and $U(R)$ be the set of all unit elements of $R$. Then $|R|=p^{n r},|Z(R)|=p^{(n-1) r}$ and $|U(R)|=p^{(n-1) r}\left(p^{r}-1\right)$ for some prime $p$ and some positive integers $n$ and $r$.

In sequel, we state two remarks which we will use throughout this paper.

Remark 3.3. Let $R_{1}$ and $R_{2}$ be two finite commutative rings with $\left|R_{1}\right|=m,\left|R_{2}\right|=n$ and $m \leq n$. It is easy to see that the subgraph of the total graph of $R_{1} \times R_{2}$ induced by the set $\{0\} \times R_{2}$ is a copy of $K_{n}$.

Remark 3.4. Let $R_{1}, R_{2}, S_{1}$ and $S_{2}$ be finite commutative rings such that $T\left(\Gamma\left(R_{1}\right)\right) \cong$ $T\left(\Gamma\left(R_{2}\right)\right.$ and $T\left(\Gamma\left(S_{1}\right)\right) \cong T\left(\Gamma\left(S_{2}\right)\right.$. Then $T\left(\Gamma\left(R_{1} \times S_{1}\right)\right) \cong T\left(\Gamma\left(R_{2} \times S_{2}\right)\right.$. However, this property does not hold in general for other widely studied graphs associated to rings (for example, the zero-divisor graphs).

Lemma 3.5. $v a\left(T\left(\Gamma\left(\mathbb{Z}_{2} \times \mathbb{Z}_{2} \times \mathbb{Z}_{2}\right)\right)\right)=v a\left(T\left(\Gamma\left(\mathbb{F}_{4} \times \mathbb{F}_{4}\right)\right)\right)=3$.

Proof. First of all, note that, in view of Remark 3.3, va(T( $\left.\left.\left(\mathbb{Z}_{2} \times \mathbb{Z}_{2} \times \mathbb{Z}_{2}\right)\right)\right)>1$. Now, we show that $\operatorname{va}\left(T\left(\Gamma\left(\mathbb{Z}_{2} \times \mathbb{Z}_{2} \times \mathbb{Z}_{2}\right)\right)\right)>2$. To this, we consider a set of vertices of the graph $T\left(\Gamma\left(\mathbb{Z}_{2} \times \mathbb{Z}_{2} \times \mathbb{Z}_{2}\right)\right)$ of the form

$$
A=\{(0,0,0),(1,0,0),(0,1,0),(0,0,1)\} .
$$

Let the set $\left\{V_{1}, V_{2}\right\}$ be an acyclic partition of $V\left(T\left(\Gamma\left(\mathbb{Z}_{2} \times \mathbb{Z}_{2} \times \mathbb{Z}_{2}\right)\right)\right)$. Since $G[A]$ is a complete graph isomorphic to $K_{4}$ and $G\left[V_{i}\right](1 \leq i \leq 2)$ have no triangle, so $\left|A \cap V_{1}\right|=$ $\left|A \cap V_{2}\right|=2$. Without the loss of generality, we may assume that $(0,0,0),(1,0,0) \in V_{1}$ and $(0,1,0),(0,0,1) \in V_{2}$. Now, consider the vertex $(0,1,1)$ of $T\left(\Gamma\left(\mathbb{Z}_{2} \times \mathbb{Z}_{2} \times \mathbb{Z}_{2}\right)\right)$. It is clear that $(0,1,1) \in V_{1}$. Therefore, each of the remaining vertex of the graph $T\left(\Gamma\left(\mathbb{Z}_{2} \times \mathbb{Z}_{2} \times \mathbb{Z}_{2}\right)\right)$ forms a triangle with two vertices of $V_{1}$. Hence, all of these vertices must be in $V_{2}$, which is a contradiction.

Now, consider the partition of $V\left(T\left(\Gamma\left(\mathbb{Z}_{2} \times \mathbb{Z}_{2} \times \mathbb{Z}_{2}\right)\right)\right)$ with sets $V_{1}=\{(0,0,0)$, $(0,1,0),(1,1,1)\}, V_{2}=\{(1,0,0),(0,0,1),(0,1,1)\}$ and $V_{3}=\{(1,0,1),(1,1,0)\}$. It is clear that the subgraphs of $T\left(\Gamma\left(\mathbb{Z}_{2} \times \mathbb{Z}_{2} \times \mathbb{Z}_{2}\right)\right)$ induced by sets $V_{1}, V_{2}$ and $V_{3}$ are acyclic. Hence $\operatorname{va}\left(T\left(\Gamma\left(\mathbb{Z}_{2} \times \mathbb{Z}_{2} \times \mathbb{Z}_{2}\right)\right)\right)=3$.

By Remark 3.3, we have $v a\left(T\left(\Gamma\left(\mathbb{F}_{4} \times \mathbb{F}_{4}\right)\right)\right)>1$. Assume that $B_{y}=\left\{(a, y): a \in \mathbb{F}_{4}\right\}$ and $C_{x}=\left\{(x, b): b \in \mathbb{F}_{4}\right\}$ for all $x, y \in \mathbb{F}_{4}$. Obviously, $\left\{B_{y}: y \in \mathbb{F}_{4}\right\}$ and $\left\{C_{x}: x \in\right.$ $\left.\mathbb{F}_{4}\right\}$ both form partitions for $V\left(T\left(\Gamma\left(\mathbb{F}_{4} \times \mathbb{F}_{4}\right)\right)\right)$. Let $\left\{V_{1}, V_{2}\right\}$ be an acyclic partition of $V\left(T\left(\Gamma\left(\mathbb{F}_{4} \times \mathbb{F}_{4}\right)\right)\right)$. Since the subgraphs of $T\left(\Gamma\left(\mathbb{F}_{4} \times \mathbb{F}_{4}\right)\right)$ induced by sets $V_{1}$ and $V_{2}$ have no triangles, each of these sets has exactly two vertices of the sets $B_{y}$ and $C_{x}$ for all 
$x, y \in \mathbb{F}_{4}$. Hence, each of the sets $V_{1}$ and $V_{2}$ has exactly two vertices such that their first components are the same and have exactly two vertices such that the second components are the same. So, each vertex in $V_{1}$ and $V_{2}$ has degree 2 , which is a contradiction, since the subgraphs of $T\left(\Gamma\left(\mathbb{F}_{4} \times \mathbb{F}_{4}\right)\right)$ induced by the sets $V_{1}$ and $V_{2}$ are union of cycles. Thus we have $v a\left(T\left(\Gamma\left(\mathbb{F}_{4} \times \mathbb{F}_{4}\right)\right)\right)>2$.

Now, according to the Figure 1, we have $\operatorname{va}\left(T\left(\Gamma\left(\mathbb{F}_{4} \times \mathbb{F}_{4}\right)\right)\right)=3$.

$(0,0)$

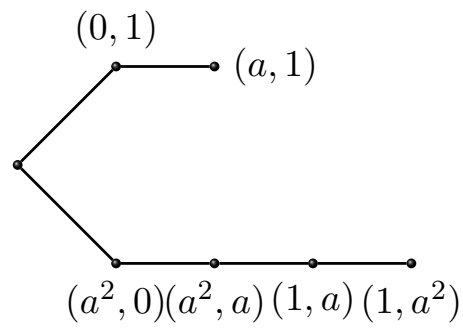

(a)

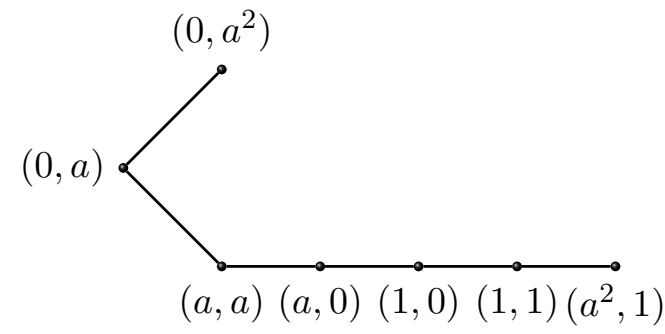

(b)

$$
\left(a, a^{2}\right) \longmapsto\left(a^{2}, a^{2}\right)
$$

(c)

Figure 1

Theorem 3.6. Let $R$ be a finite commutative ring such that $v a(T(\Gamma(R)))=2$. Then the following statements hold.

(i) If $R$ is local, then $R$ is isomorphic to one of the following rings: $\mathbb{Z}_{9}, \frac{\mathbb{Z}_{3}[x]}{\left(x^{2}\right)}, \mathbb{Z}_{8}, \frac{\mathbb{Z}_{2}[x]}{\left(x^{3}\right)}, \frac{\mathbb{Z}_{4}[x]}{\left(2 x, x^{2}-2\right)}, \frac{\mathbb{Z}_{2}[x, y]}{(x, y)^{2}}, \frac{\mathbb{Z}_{4}[x]}{(2, x)^{2}}, \frac{\mathbb{F}_{4}[x]}{\left(x^{2}\right)}, \frac{\mathbb{Z}_{4}[x]}{\left(x^{2}+x+1\right)}$

(ii) If $R$ is not local, then $R$ is isomorphic to one of the following rings: $\mathbb{Z}_{2} \times \mathbb{Z}_{2}, \mathbb{Z}_{6}, \mathbb{Z}_{2} \times \mathbb{Z}_{4}, \mathbb{Z}_{2} \times \frac{\mathbb{Z}_{2}[x]}{\left(x^{2}\right)}, \mathbb{Z}_{2} \times \mathbb{F}_{4}, \mathbb{Z}_{3} \times \mathbb{Z}_{3}, \mathbb{Z}_{3} \times \mathbb{F}_{4}$

Proof. (i) Assume that $R$ is a local ring, and let $|Z(R)|=n$ and $\left|\frac{R}{Z(R)}\right|=m$. Then by Theorem 2.5, $T(\Gamma(R))$ has an induced subgraph isomorphic to $K_{n}$ and so by Remark 2.2, $|Z(R)| \leq 4$. Now, we consider the following two cases:

(a) If $2 \in Z(R)$, then by Theorem $3.2,|R|=2^{k}$ and $k \leq 4$. Since $v a(T(\Gamma(R)))=2$, Theorem 3.1 implies that $|R|=16,8$. According to Corbas and Williams [6] there are two non-isomorphic rings of order 16 with maximal ideals of order 4 , namely $\frac{\mathbb{F}_{4}[x]}{\left(x^{2}\right)}$ and $\frac{\mathbb{Z}_{4}[x]}{\left(x^{2}+x+1\right)}$ (see also Redmond [11]), so for these rings have $T(\Gamma(R)) \cong 4 K_{4}$. Therefore, by Remark 2.2, these rings have vertex-arboricity 2. In [6] it is also shown that there are 5 local rings of order 8 (except $\mathbb{F}_{8}$ ) as follows:

$$
\mathbb{Z}_{8}, \frac{\mathbb{Z}_{2}[x]}{\left(x^{3}\right)}, \frac{\mathbb{Z}_{4}[x]}{\left(2 x, x^{2}-2\right)}, \frac{\mathbb{Z}_{2}[x, y]}{(x, y)^{2}}, \frac{\mathbb{Z}_{4}[x]}{(2, x)^{2}} .
$$

In all of these rings we have $|Z(R)|=4$ and hence $T(\Gamma(R)) \cong 2 K_{4}$. Then, by Remark 2.2 , these rings have vertex-arboricity 2 .

(b) If $2 \notin Z(R)$, then $|Z(R)|=3$. According to [6], there are two rings of order 9 namely, $\mathbb{Z}_{9}$ and $\frac{\mathbb{Z}_{3}[x]}{\left(x^{2}\right)}$. For these rings, we have $T(\Gamma(R)) \cong K_{3} \cup K_{3,3}$. Hence, by Corollary 2.7 , these rings have vertex-arboricity 2 .

(ii) Suppose that $R$ is not local. Since $R$ is finite, there are finite local rings $R_{1}, \ldots, R_{t}$ (with $t \geq 2$ ) such that $R=R_{1} \times R_{2} \times \cdots \times R_{t}$. Now, according to Remarks 2.2 and 3.3, 
we have the following candidates:

$\mathbb{Z}_{2} \times \mathbb{Z}_{2}, \mathbb{Z}_{6}, \mathbb{Z}_{2} \times \mathbb{Z}_{4}, \mathbb{Z}_{2} \times \frac{\mathbb{Z}_{2}[x]}{\left(x^{2}\right)}, \mathbb{Z}_{2} \times \mathbb{F}_{4}, \mathbb{Z}_{3} \times \mathbb{Z}_{3}, \mathbb{Z}_{3} \times \mathbb{Z}_{4}, \mathbb{Z}_{3} \times \frac{\mathbb{Z}_{2}[x]}{\left(x^{2}\right)}, \mathbb{Z}_{3} \times \mathbb{F}_{4}$,

$\mathbb{Z}_{2} \times \mathbb{Z}_{2} \times \mathbb{Z}_{2}, \mathbb{Z}_{4} \times \mathbb{Z}_{4}, \mathbb{Z}_{4} \times \frac{\mathbb{Z}_{2}[x]}{\left(x^{2}\right)}, \frac{\mathbb{Z}_{2}[x]}{\left(x^{2}\right)} \times \frac{\mathbb{Z}_{2}[x]}{\left(x^{2}\right)}, \mathbb{Z}_{4} \times \mathbb{F}_{4}, \frac{\mathbb{Z}_{2}[x]}{\left(x^{2}\right)} \times \mathbb{F}_{4}, \mathbb{F}_{4} \times \mathbb{F}_{4}$

Now we examine each of the above rings.

The total graph of the ring $\mathbb{Z}_{2} \times \mathbb{Z}_{2}$ is isomorphic to the cycle of size 4 . We consider the acyclic partition $V_{1}=\{(0,0),(1,0)\}$ and $V_{2}=\{(0,1),(1,1)\}$ of $V\left(T\left(\Gamma\left(\mathbb{Z}_{2} \times \mathbb{Z}_{2}\right)\right)\right.$ ). Hence, the subgraphs of $T\left(\Gamma\left(\mathbb{Z}_{2} \times \mathbb{Z}_{2}\right)\right)$ induced by sets $V_{1}$ and $V_{2}$ are acyclic. Thus $v a\left(T\left(\Gamma\left(\mathbb{Z}_{2} \times \mathbb{Z}_{2}\right)\right)\right)=2$.

For $\mathbb{Z}_{6}$, by considering the acyclic partition $V_{1}=\{0,1,3\}$ and $V_{2}=\{2,4,6\}$ of $V\left(T\left(\Gamma\left(\mathbb{Z}_{6}\right)\right)\right)$, we have $v a\left(T\left(\Gamma\left(\mathbb{Z}_{6}\right)\right)\right)=2$.

For $\mathbb{Z}_{2} \times \mathbb{Z}_{4}$, we put $V_{1}=\{(0,0),(0,2),(1,1),(1,3)\}$ and $V_{2}=\{(0,1),(0,3),(1,0),(1,2)\}$. Now, it is easy to see that $v a\left(T\left(\Gamma\left(\mathbb{Z}_{2} \times \mathbb{Z}_{4}\right)\right)\right)=2$. Since $T\left(\Gamma\left(\mathbb{Z}_{4}\right)\right) \cong T\left(\Gamma\left(\frac{\mathbb{Z}_{2}[x]}{\left(x^{2}\right)}\right)\right)$, by Remark 3.4, we have $T\left(\Gamma\left(\mathbb{Z}_{2} \times \mathbb{Z}_{4}\right)\right) \cong T\left(\Gamma\left(\mathbb{Z}_{2} \times \frac{\mathbb{Z}_{2}[x]}{\left(x^{2}\right)}\right)\right)$. Thus $v a\left(T\left(\Gamma\left(\mathbb{Z}_{2} \times \frac{\mathbb{Z}_{2}[x]}{\left(x^{2}\right)}\right)\right)\right)=2$.

For $\mathbb{Z}_{2} \times \mathbb{F}_{4}$, by using the acyclic partition

$$
V_{1}=\{(0,0),(0,1),(1,0),(1, a)\} \text { and } V_{2}=\left\{(0, a),\left(0, a^{2}\right),(1,1),\left(1, a^{2}\right)\right\}
$$

of $V\left(T\left(\Gamma\left(\mathbb{Z}_{2} \times \mathbb{F}_{4}\right)\right)\right)$, we have $v a\left(T\left(\Gamma\left(\mathbb{Z}_{2} \times \mathbb{F}_{4}\right)\right)\right)=2$.

For $\mathbb{Z}_{3} \times \mathbb{Z}_{3}$, we consider the acyclic partition $V_{1}=\{(0,0),(0,1),(1,0),(1,1),(2,1)\}$ and $V_{2}=\{(0,2),(2,0),(1,2),(2,2)\}$ of $V\left(T\left(\Gamma\left(\mathbb{Z}_{3} \times \mathbb{Z}_{3}\right)\right)\right)$. Hence $v a\left(T\left(\Gamma\left(\mathbb{Z}_{3} \times \mathbb{Z}_{3}\right)\right)\right)=2$.

For $\mathbb{Z}_{3} \times \mathbb{Z}_{4}$, the graph $T\left(\Gamma\left(\mathbb{Z}_{3} \times \mathbb{Z}_{4}\right)\right)$ has a complete graph $K_{6}$ as a subgraph with vertex set $\{(0,0),(1,0),(2,0),(0,2),(1,2),(2,2)\}$, and so, by Remark 2.2 , we have $v a\left(T\left(\Gamma\left(\mathbb{Z}_{3} \times\right.\right.\right.$ $\left.\left.\left.\mathbb{Z}_{4}\right)\right)\right)>2$. Also by Remark 3.4 , we have $T\left(\Gamma\left(\mathbb{Z}_{3} \times \mathbb{Z}_{4}\right)\right) \cong T\left(\Gamma\left(\mathbb{Z}_{3} \times \frac{\mathbb{Z}_{2}[x]}{\left(x^{2}\right)}\right)\right)$. Thus $v a\left(T\left(\Gamma\left(\mathbb{Z}_{3} \times \frac{\mathbb{Z}_{2}[x]}{\left(x^{2}\right)}\right)\right)\right)>2$.

For $\mathbb{Z}_{3} \times \mathbb{F}_{4}$, according to the Figure 2 we have $v a\left(T\left(\Gamma\left(\mathbb{Z}_{3} \times \mathbb{F}_{4}\right)\right)\right)=2$.

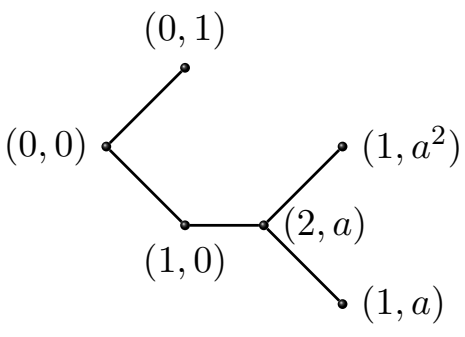

(a)

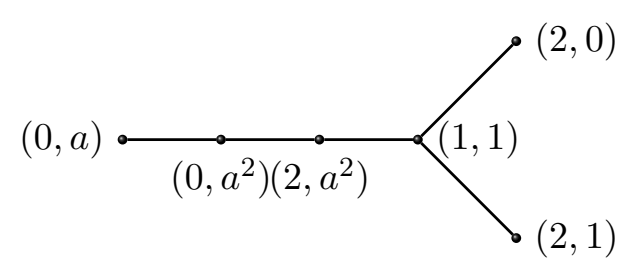

(b)

Figure 2

For $\mathbb{Z}_{2} \times \mathbb{Z}_{2} \times \mathbb{Z}_{2}$, by Lemma 3.5 , we have $v a\left(T\left(\Gamma\left(\mathbb{Z}_{2} \times \mathbb{Z}_{2} \times \mathbb{Z}_{2}\right)\right)\right)>2$.

For $\mathbb{Z}_{4} \times \mathbb{Z}_{4}$, the graph $T\left(\Gamma\left(\mathbb{Z}_{4} \times \mathbb{Z}_{4}\right)\right)$ has a $K_{8}$ as a subgraph with vertex set

$$
\{(0,0),(1,0),(2,0),(3,0),(0,2),(1,2),(2,2),(3,2)\},
$$

and so, by Remark 2.2 , we have $v a\left(T\left(\Gamma\left(\mathbb{Z}_{4} \times \mathbb{Z}_{4}\right)\right)\right)>3$.

According to Remark 3.4, $T\left(\Gamma\left(\mathbb{Z}_{4} \times \mathbb{Z}_{4}\right)\right) \cong T\left(\Gamma\left(\mathbb{Z}_{4} \times \frac{\mathbb{Z}_{2}[x]}{\left(x^{2}\right)}\right)\right) \cong T\left(\Gamma\left(\frac{\mathbb{Z}_{2}[x]}{\left(x^{2}\right)} \times \frac{\mathbb{Z}_{2}[x]}{\left(x^{2}\right)}\right)\right)$. So the vertex-arboricity of graphs $T\left(\Gamma\left(\mathbb{Z}_{4} \times \frac{\mathbb{Z}_{2}[x]}{\left(x^{2}\right)}\right)\right)$ and $T\left(\Gamma\left(\frac{\mathbb{Z}_{2}[x]}{\left(x^{2}\right)} \times \frac{\mathbb{Z}_{2}[x]}{\left(x^{2}\right)}\right)\right)$ is greater than three.

For $\mathbb{Z}_{4} \times \mathbb{F}_{4}$, the graph $T\left(\Gamma\left(\mathbb{Z}_{4} \times \mathbb{F}_{4}\right)\right)$ has a $K_{8}$ as a subgraph with vertex set

$$
\left\{(0,0),(0,1),(0, a),\left(0, a^{2}\right),(2,0),(2,1),(2, a),\left(2, a^{2}\right)\right\},
$$


and so, by Remark 2.2 , we have $v a\left(T\left(\Gamma\left(\mathbb{Z}_{4} \times \mathbb{F}_{4}\right)\right)\right)>3$. Also by Remark 3.4, $T\left(\Gamma\left(\mathbb{Z}_{4} \times\right.\right.$ $\left.\left.\mathbb{F}_{4}\right)\right) \cong T\left(\Gamma\left(\frac{\mathbb{Z}_{2}[x]}{\left(x^{2}\right)} \times \mathbb{F}_{4}\right)\right)$. Therefore $v a\left(T\left(\Gamma\left(\frac{\mathbb{Z}_{2}[x]}{\left(x^{2}\right)} \times \mathbb{F}_{4}\right)\right)\right)>3$.

For $\mathbb{F}_{4} \times \mathbb{F}_{4}$, by Lemma 3.5 , we have $v a\left(T\left(\Gamma\left(\mathbb{F}_{4} \times \mathbb{F}_{4}\right)\right)\right)>2$.

Lemma 3.7. For the ring $\mathbb{Z}_{2} \times \mathbb{Z}_{2} \times \mathbb{Z}_{3}$, va $\left(T\left(\Gamma\left(\mathbb{Z}_{2} \times \mathbb{Z}_{2} \times \mathbb{Z}_{3}\right)\right)\right)=4$.

Proof. First, by Remark 3.3, we have $v a\left(T\left(\Gamma\left(\mathbb{Z}_{2} \times \mathbb{Z}_{2} \times \mathbb{Z}_{3}\right)\right)\right)>2$.

Now, let $T\left(\Gamma\left(\mathbb{Z}_{2} \times \mathbb{Z}_{2} \times \mathbb{Z}_{3}\right)\right)=G$ and $A=A_{0} \cup A_{1}$, where $A_{0}=\left\{(0,0, z): z \in \mathbb{Z}_{3}\right\}$ and $A_{1}=\left\{(0,1, z): z \in \mathbb{Z}_{3}\right\}$. Also put $B=B_{0} \cup B_{1}$, where $B_{0}=\left\{(1,0, z): z \in \mathbb{Z}_{3}\right\}$ and $B_{1}=\left\{(1,1, z): z \in \mathbb{Z}_{3}\right\}$. It is clear that the two sets $A$ and $B$ are partition for $V(G)$. Let $\left\{V_{1}, V_{2}, V_{3}\right\}$ be an acyclic partition for $V(G)$. If $\left|V_{j}\right| \geq 5$ for some $j \in\{1,2,3\}$, then $\left|A \cap V_{j}\right| \geq 3$ or $\left|B \cap V_{j}\right| \geq 3$, which is impossible, since $G[A]$ and $G[B]$ are complete graphs isomorphic to $K_{6}$ and $G\left[V_{i}\right](1 \leq i \leq 3)$ are acyclic induced subgraphs of $G$. Therefore $\left|V_{i}\right|=4$ for some $i \in\{1,2,3\}$.

We know that every vertex of $G\left[A_{0}\right]\left(G\left[A_{1}\right]\right)$ are adjacent to every vertex of $G\left[B_{0}\right]$ $\left(G\left[B_{1}\right]\right)$ and $G\left[V_{i}\right](1 \leq i \leq 3)$ are acyclic induced subgraphs of $G$. Hence without the loss of generality we can assume that $\left|A_{0} \cap V_{1}\right|=\left|B_{1} \cap V_{1}\right|=2$ and $\left|A_{1} \cap V_{2}\right|=\left|B_{0} \cap V_{2}\right|=2$. Then $V_{3}=\left\{a_{0}, a_{1}, b_{0}, b_{1}: a_{s} \in A_{s}, b_{t} \in B_{t}, 0 \leq s, t \leq 1\right\}$. It follows that $G\left[V_{3}\right]$ is a cycle of length 4 , which is a contradiction and so $v a(G)>3$.

Now, by using the following partition of $V(G)$, we have that $v a(G)=4$.

$$
\begin{array}{ll}
V_{1}=\{(0,0,0),(1,0,0),(1,1,2)\}, & V_{2}=\{(0,1,0),(1,1,1),(1,0,1)\}, \\
V_{3}=\{(0,1,2),(0,0,2),(1,0,2)\}, & V_{4}=\{(0,0,1),(0,1,1),(1,1,0)\} .
\end{array}
$$

Theorem 3.8. Let $R$ be a finite commutative ring such that $v a(T(\Gamma(R)))=3$. Then the following statements hold.

(i) If $R$ is local, then $R$ is isomorphic to $\mathbb{Z}_{25}$ or $\frac{\mathbb{Z}_{5}[x]}{\left(x^{2}\right)}$.

(ii) If $R$ is not local, then $R$ is isomorphic to one of the following rings:

$\mathbb{Z}_{3} \times \mathbb{Z}_{4}, \mathbb{Z}_{3} \times \frac{\mathbb{Z}_{2}[x]}{\left(x^{2}\right)}, \mathbb{Z}_{2} \times \mathbb{Z}_{2} \times \mathbb{Z}_{2}, \mathbb{F}_{4} \times \mathbb{F}_{4}, \mathbb{Z}_{2} \times \mathbb{Z}_{5}, \mathbb{Z}_{3} \times \mathbb{Z}_{5}, \mathbb{F}_{4} \times \mathbb{Z}_{5}, \mathbb{Z}_{5} \times \mathbb{Z}_{5}$

Proof. (i) Assume that $R$ is a local ring. We consider the following two cases:

(a) If $2 \in Z(R)$, then, by Theorem 2.5 , we have $T(\Gamma(R)) \cong m K_{n}$. Hence, by Remark $2.2,5 \leq|Z(R)| \leq 6$. But, in this situation $2 \in Z(R)$, and so, there are no such local rings.

(b) If $2 \notin Z(R)$, then, by Theorem 2.5 , we have $T(\Gamma(R)) \cong K_{n} \bigcup\left(\frac{m-1}{2}\right) K_{n, n}$. Hence, by Remark $2.2,5 \leq|Z(R)| \leq 6$. Therefore $|Z(R)|=5$ and so there exist two local rings, $\mathbb{Z}_{25}$ and $\frac{\mathbb{Z}_{5}[x]}{\left(x^{2}\right)}$ of order 25 . For these rings we have $T(\Gamma(R)) \cong K_{5} \cup 2 K_{5,5}$. Hence, by Corollary 2.7, we have $v a(T(\Gamma(R)))=3$.

(ii) Suppose that $R$ is not a local ring. Arguments similar to those used in proof of Theorem 3.6 (ii), in conjunction with Remarks 2.2 and 3.3 show that we have the following candidates:

$\mathbb{Z}_{2} \times \mathbb{Z}_{2}, \mathbb{Z}_{6}, \mathbb{Z}_{2} \times \mathbb{Z}_{4}, \mathbb{Z}_{2} \times \frac{\mathbb{Z}_{2}[x]}{\left(x^{2}\right)}, \mathbb{Z}_{2} \times \mathbb{F}_{4}, \mathbb{Z}_{3} \times \mathbb{Z}_{3}, \mathbb{Z}_{3} \times \mathbb{Z}_{4}, \mathbb{Z}_{3} \times \frac{\mathbb{Z}_{2}[x]}{\left(x^{2}\right)}, \mathbb{Z}_{3} \times \mathbb{F}_{4}$,

$\mathbb{Z}_{2} \times \mathbb{Z}_{2} \times \mathbb{Z}_{2}, \mathbb{Z}_{4} \times \mathbb{Z}_{4}, \mathbb{Z}_{4} \times \frac{\mathbb{Z}_{2}[x]}{\left(x^{2}\right)}, \frac{\mathbb{Z}_{2}[x]}{\left(x^{2}\right)} \times \frac{\mathbb{Z}_{2}[x]}{\left(x^{2}\right)}, \mathbb{Z}_{4} \times \mathbb{F}_{4}, \frac{\mathbb{Z}_{2}[x]}{\left(x^{2}\right)} \times \mathbb{F}_{4}, \mathbb{F}_{4} \times \mathbb{F}_{4}$,

$\mathbb{Z}_{2} \times \mathbb{Z}_{5}, \mathbb{Z}_{2} \times \mathbb{Z}_{2} \times \mathbb{Z}_{3}, \mathbb{Z}_{3} \times \mathbb{Z}_{5}, \mathbb{Z}_{4} \times \mathbb{Z}_{5}, \frac{\mathbb{Z}_{2}[x]}{\left(x^{2}\right)} \times \mathbb{Z}_{5}, \mathbb{F}_{4} \times \mathbb{Z}_{5}, \mathbb{Z}_{5} \times \mathbb{Z}_{5}$

According to the proof of Theorem 3.6 (ii), we examine the following cases:

For $\mathbb{Z}_{3} \times \mathbb{Z}_{4}$, we consider the partition

$$
\begin{aligned}
& V_{1}=\{(0,0),(1,1),(1,2),(1,3)\}, \\
& V_{2}=\{(0,2),(2,0),(2,1),(2,3)\}
\end{aligned}
$$

and

$$
V_{3}=\{(0,1),(0,3),(1,0),(2,2)\}
$$


of $V\left(T\left(\Gamma\left(\mathbb{Z}_{3} \times \mathbb{Z}_{4}\right)\right)\right)$. The subgraphs of $T\left(\Gamma\left(\mathbb{Z}_{3} \times \mathbb{Z}_{4}\right)\right)$ induced by the sets $V_{1}, V_{2}$ and $V_{3}$ are acyclic graphs. Hence, we have $v a\left(T\left(\Gamma\left(\mathbb{Z}_{3} \times \mathbb{Z}_{4}\right)\right)\right)=3$. The Remark 3.4 implies that $T\left(\Gamma\left(\mathbb{Z}_{3} \times \mathbb{Z}_{4}\right)\right) \cong T\left(\Gamma\left(\mathbb{Z}_{3} \times \frac{\mathbb{Z}_{2}[x]}{\left(x^{2}\right)}\right)\right)$ and so $v a\left(T\left(\Gamma\left(\mathbb{Z}_{3} \times \frac{\mathbb{Z}_{2}[x]}{\left(x^{2}\right)}\right)\right)\right)=3$.

For rings $\mathbb{Z}_{2} \times \mathbb{Z}_{2} \times \mathbb{Z}_{2}$ and $\mathbb{F}_{4} \times \mathbb{F}_{4}$, by Lemma 3.5 , we have $v a\left(T\left(\Gamma\left(\mathbb{Z}_{2} \times \mathbb{Z}_{2} \times \mathbb{Z}_{2}\right)\right)\right)=$ $\operatorname{va}\left(T\left(\Gamma\left(\mathbb{F}_{4} \times \mathbb{F}_{4}\right)\right)\right)=3$.

For $\mathbb{Z}_{2} \times \mathbb{Z}_{5}$, consider the acyclic partition $V_{1}=\{(0,0),(0,1),(1,1),(1,2)\}, V_{2}=\{(0,2)$, $(0,3),(1,0),(1,4)\}$ and $V_{3}=\{(0,4),(1,3)\}$ of $V\left(T\left(\Gamma\left(\mathbb{Z}_{2} \times \mathbb{Z}_{5}\right)\right)\right)$. Now, it is easy to see that $\operatorname{va}\left(T\left(\Gamma\left(\mathbb{Z}_{2} \times \mathbb{Z}_{5}\right)\right)\right)=3$.

For $\mathbb{Z}_{2} \times \mathbb{Z}_{2} \times \mathbb{Z}_{3}$, by Lemma 3.7 , we have $v a\left(T\left(\Gamma\left(\mathbb{Z}_{2} \times \mathbb{Z}_{2} \times \mathbb{Z}_{3}\right)\right)\right)>3$.

For $\mathbb{Z}_{3} \times \mathbb{Z}_{5}$, by using the acyclic partition

$$
\begin{gathered}
V_{1}=\{(0,4),(1,0),(1,3),(2,3)\}, \\
V_{2}=\{(0,0),(0,1),(1,2),(1,4),(2,1)\}
\end{gathered}
$$

and

$$
V_{3}=\{(0,2),(0,3),(1,1),(2,0),(2,2),(2,4)\}
$$

of $V\left(T\left(\Gamma\left(\mathbb{Z}_{3} \times \mathbb{Z}_{5}\right)\right)\right)$, we have $v a\left(T\left(\Gamma\left(\mathbb{Z}_{3} \times \mathbb{Z}_{5}\right)\right)\right)=3$.

For $\mathbb{Z}_{4} \times \mathbb{Z}_{5}$, the graph $T\left(\Gamma\left(\mathbb{Z}_{4} \times \mathbb{Z}_{5}\right)\right)$ has a complete graph $K_{10}$ as a subgraph with vertex set $\{(0,0),(0,1),(0,2),(0,3),(0,4),(2,0),(2,1),(2,2),(2,3),(2,4)\}$, and so, we have $v a\left(T\left(\Gamma\left(\mathbb{Z}_{4} \times \mathbb{Z}_{5}\right)\right)\right) \geq 5$. Also, Remark 3.4, $T\left(\Gamma\left(\mathbb{Z}_{4} \times \mathbb{Z}_{5}\right)\right) \cong T\left(\Gamma\left(\frac{\mathbb{Z}_{2}[x]}{\left(x^{2}\right)} \times \mathbb{Z}_{5}\right)\right)$ and so $\operatorname{va}\left(T\left(\Gamma\left(\frac{\mathbb{Z}_{2}[x]}{\left(x^{2}\right)} \times \mathbb{Z}_{5}\right)\right)\right) \geq 5$.

For $\mathbb{F}_{4} \times \mathbb{Z}_{5}$, according to Figure 3 , we have $\operatorname{va}\left(T\left(\Gamma\left(\mathbb{F}_{4} \times \mathbb{Z}_{5}\right)\right)\right)=3$.

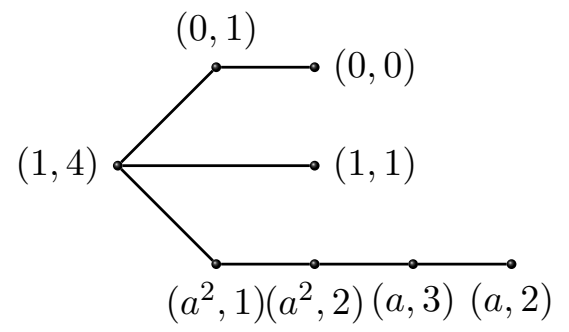

(a)

$(0,2)$

$$
(0,3)(1,2)(1,0)(a, 0)(a, 1)
$$

(b)

$$
\left(a^{2}, 0\right)\left(a^{2}, 3\right)(\stackrel{\bullet}{\bullet}, \stackrel{\bullet}{\bullet}(1,3)(\stackrel{\bullet}{a}, 4)
$$

(c)

\section{Figure 3}

For $\mathbb{Z}_{5} \times \mathbb{Z}_{5}$, by Figure 4 , we conclude that $\operatorname{va}\left(T\left(\Gamma\left(\mathbb{Z}_{5} \times \mathbb{Z}_{5}\right)\right)\right)=3$.

Thus the proof is complete.

\section{The arboricity of the total graph}

In this section, we characterize all finite commutative rings whose total graph has arboricity two or three. In addition, we show that, for a positive integer $v$, there are only finitely many finite rings whose total graph has arboricity $v$. We begin the section with the following result of C. St. J. A. Nash-Williams.

Theorem $4.1([9])$. For a graph $G, \nu(G)=\max \left\lceil\frac{e_{H}}{n_{H}-1}\right\rceil$, where $n_{H}=|V(H)|, e_{H}=$ $|E(H)|$ and $H$ ranges over all non-trivial induced subgraphs of $G$. 


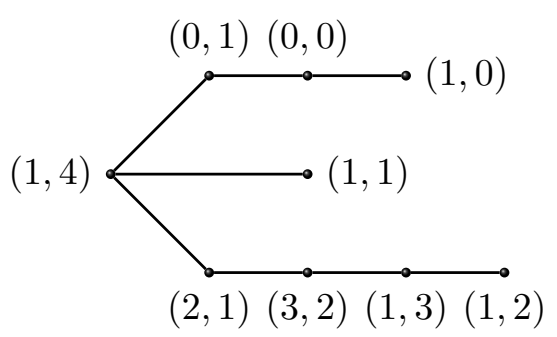

(a)
$(3,1)$

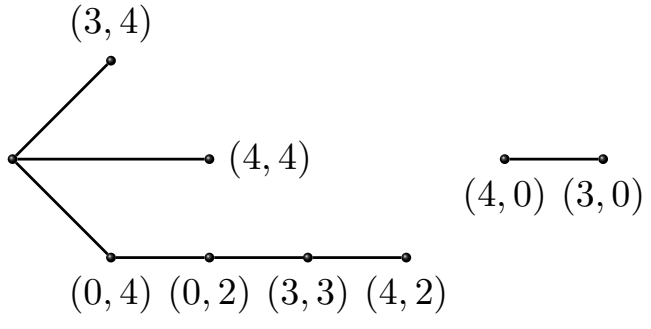

(b)

$(0,3)$

$(2,2)$

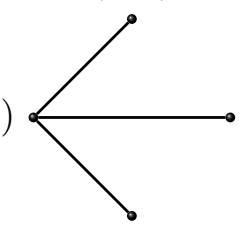

$(4,3)$ $(\stackrel{2,4)(4,1)}{(2,0)}$

(c)

Figure 4

Theorem 4.2. For a graph $G,\left\lceil\frac{\delta(G)+1}{2}\right\rceil \leq \nu(G) \leq\left\lceil\frac{\Delta(G)+1}{2}\right\rceil$. In particular, if $G$ is $d$-regular, then $\nu(G)=\left\lceil\frac{d+1}{2}\right\rceil=\left\lceil\frac{e}{n-1}\right\rceil$, where $n=|V(G)|$ and $e=|E(G)|$.

Proof. First, it is clear that, if $G$ has some isolated vertices, say $X=\left\{x_{1}, x_{2}, \ldots, x_{k}\right\}$, then $\nu(G)=\nu(G[V(G) \backslash X])$. So, we can assume that $G$ has no isolated vertices. Let $H$ be a subgraph of $G$ with $|V(H)|=n^{\prime}$ and $|E(H)|=e^{\prime}$. Then we have

$$
\frac{e^{\prime}}{n^{\prime}-1} \leq \frac{\Delta(H) n^{\prime}}{2\left(n^{\prime}-1\right)}=\frac{1}{2}\left(\Delta(H)+\frac{\Delta(H)}{n^{\prime}-1}\right) .
$$

Since $\Delta(H) \leq \min \left\{\Delta(G), n^{\prime}-1\right\}$, we have $\frac{e^{\prime}}{n^{\prime}-1} \leq \frac{\Delta(G)+1}{2}$, and hence, by Theorem 4.1, $\nu(G) \leq\left\lceil\frac{\Delta(G)+1}{2}\right\rceil$. On the other hand $\frac{e}{n-1} \geq \frac{\delta(G) n}{2(n-1)}>\frac{\delta(G)}{2}$. Since $\nu(G)$ is an integer, $\nu(G) \geq\left\lceil\frac{\delta(G)+1}{2}\right\rceil$, as required.

Clearly, in view of the above theorem, $\nu\left(K_{n}\right)=\left\lceil\frac{n}{2}\right\rceil$. So, by arguing as in the proof of Theorem 2.4, we have the following theorem.

Theorem 4.3. For any positive integer $v$, the number of finite rings $R$ whose total graph has arboricity $v$ is finite.

Theorem 3.1 implies that $T(\Gamma(R))$ has arboricity one if and only if either $R$ is an integral domain or $R$ is isomorphic to $\mathbb{Z}_{4}$ or $\frac{\mathbb{Z}_{2}[x]}{\left(x^{2}\right)}$. Now, we will classify, up to isomorphism, all the finite commutative rings whose total graph has arboricity two or three.

Theorem 4.4. Let $R$ be a finite ring such that $\nu(T(\Gamma(R)))=2$. Then the following statements hold.

(i) If $R$ is local, then $R$ is isomorphic to one of the following rings:

$$
\mathbb{Z}_{9}, \frac{\mathbb{Z}_{3}[x]}{\left(x^{2}\right)}, \mathbb{Z}_{8}, \frac{\mathbb{Z}_{2}[x]}{\left(x^{3}\right)}, \frac{\mathbb{Z}_{4}[x]}{\left(2 x, x^{2}-2\right)}, \frac{\mathbb{Z}_{2}[x, y]}{(x, y)^{2}}, \frac{\mathbb{Z}_{4}[x]}{(2, x)^{2}}, \frac{\mathbb{F}_{4}[x]}{\left(x^{2}\right)}, \frac{\mathbb{Z}_{4}[x]}{\left(x^{2}+x+1\right)} .
$$

(ii) If $R$ is not local, then $R$ is isomorphic to $\mathbb{Z}_{2} \times \mathbb{Z}_{2}$ or $\mathbb{Z}_{6}$.

Proof. (i) Assume that $R$ is a local ring. If $2 \in Z(R)$, then, by Lemma 2.1 and Theorem 4.2 , we have $|Z(R)|=4$. Then by Theorem $3.2,|R|=16,8$. Now, by same argument of 
Theorem 3.6, $R$ is isomorphic to one of the following rings:

$$
\mathbb{Z}_{8}, \frac{\mathbb{Z}_{2}[x]}{\left(x^{3}\right)}, \frac{\mathbb{Z}_{4}[x]}{\left(2 x, x^{2}-2\right)}, \frac{\mathbb{Z}_{2}[x, y]}{(x, y)^{2}}, \frac{\mathbb{Z}_{4}[x]}{(2, x)^{2}}, \frac{\mathbb{F}_{4}[x]}{\left(x^{2}\right)}, \frac{\mathbb{Z}_{4}[x]}{\left(x^{2}+x+1\right)} .
$$

If $2 \notin Z(R)$, then $|Z(R)|=3$. So, $R$ is isomorphic to $\mathbb{Z}_{9}$ or $\frac{\mathbb{Z}_{3}[x]}{\left(x^{2}\right)}$.

(ii) If $R$ is not a local ring, then, by Theorem 4.2 , we have $3 \leq|Z(R)| \leq 4$. When $|Z(R)|=3$, it is clear that $R$ is isomorphic to $\mathbb{Z}_{2} \times \mathbb{Z}_{2}$. Moreover, if $|Z(R)|=4$, then $R$ is isomorphic to $\mathbb{Z}_{6}$, and so the proof is complete.

By slight modifications in the proof of Theorem 4.4, one can prove the following theorem.

Theorem 4.5. Let $R$ be a finite ring such that $\nu(T(\Gamma(R)))=3$. Then the following statements hold.

(i) If $R$ is local, then $R$ is isomorphic to $\mathbb{Z}_{25}$ or $\frac{\mathbb{Z}_{5}[x]}{\left(x^{2}\right)}$.

(ii) If $R$ is not local, then $R$ is isomorphic to one of the following rings:

$$
\mathbb{Z}_{2} \times \mathbb{F}_{4}, \mathbb{Z}_{3} \times \mathbb{Z}_{3}, \mathbb{Z}_{2} \times \mathbb{Z}_{4}, \mathbb{Z}_{2} \times \frac{\mathbb{Z}_{2}[x]}{\left(x^{2}\right)}, \mathbb{Z}_{2} \times \mathbb{Z}_{5}, \mathbb{Z}_{3} \times \mathbb{F}_{4}
$$

In general, we can determine the arboricity of the total graph as in the following theorem.

Theorem 4.6. Let $R$ be a finite ring.

(i) If $2 \in Z(R)$, then $\nu(T(\Gamma(R)))=\left\lceil\frac{|Z(R)|}{2}\right\rceil$.

(ii) If $2 \notin Z(R)$, then the following statements hold.

(1) If $|Z(R)|=2 k+1$, then $\nu(T(\Gamma(R)))=k+1$.

(2) If $|Z(R)|=2 k$, then $k \leq \nu(T(\Gamma(R))) \leq k+1$.

Proof. It follows from Lemma 2.1 and Theorem 4.2.

\section{References}

[1] D.F. Anderson and A. Badawi, The total graph of a commutative ring, J. Algebra, 320, 2706-2719, 2008.

[2] D.F. Anderson and A. Badawi, The total graph of a commutative ring without the zero element, J. Algebra Appl. 11 1-18 pages, 2012.

[3] G.J. Chang, C. Chen and Y. Chen, Vertex and tree arboricities of graphs, J. Comb. Optim. 8 295-306, 2004.

[4] G. Chartrand, H.V. Kronk and C.E. Wall, The point arboricity of a graph, Israel J. Math. 6, 169-175, 1968.

[5] T.T. Chelvam and T. Asir, On the genus of the total graph of a commutative ring, Comm. Algebra, 41, 142-153, 2013.

[6] B. Corbas and G.D. Williams, Ring of order p5. II. Local rings, J. Algebra, 231 (2), 691-704, 2000.

[7] H.R. Maimani, C. Wickham and S. Yassemi, Rings whose total graph have genus at most one, Rocky Mountain J. Math. 42, 1551-1560, 2012.

[8] B.R. McDonald, Finite rings with identity , Pure Appl. Math. 28, Marcel Dekker, Inc., New York, 1974.

[9] C.St.J.A. Nash-Williams, Decomposition of finite graphs into forests, Journal London Math. Soc, 39, 12, 1964.

[10] R. Raghavendran, iFinite associative rings, Compositio Math. 21, 195-229, 1969.

[11] S.P. Redmond, On zero-divisor graphs of small finite commutative rings, Discrete Math. 307, 1155-1166, 2007. 